\title{
Kinetics of Contact Electrification Between Metals and Polymers.
}

\author{
Bartosz A. Grzybowski*, Marcin Fialkowski, Jason A. Wiles \\ Department of Chemical and Biological Engineering \\ Northwestern University \\ 2145 Sheridan Rd. \\ Evanston, IL 60208, USA.
}

\section{Supporting Information}

In a set of independent experiments we verified that an increase in the compressive force between the metal and the polymer surfaces does indeed give rise to more charge separation between them. Our experimental arrangement (Fig. S1) comprised two long, steel cylinders sandwiched between two polystyrene plates, as well as a metal electrode positioned below the bottom plate along the direction perpendicular to the long axes of the cylinders and connected to an electrometer. When the upper surface was periodically moved back-and-forth causing cylinders to roll, these cylinders developed positive charge; when one of them moved above the electrode, its charge was sensed by capacitative coupling. The magnitude of the developed charge, $Q$, increased linearly with a mechanical force, $F$, applied to the upper plate (by putting $m=10-50 \mathrm{~g}$ weights onto it). Since for the cylinder pressed against a compliant surface, the area of contact scales linearly with the applied force, it follows that for this experimental geometry $A \propto Q$.

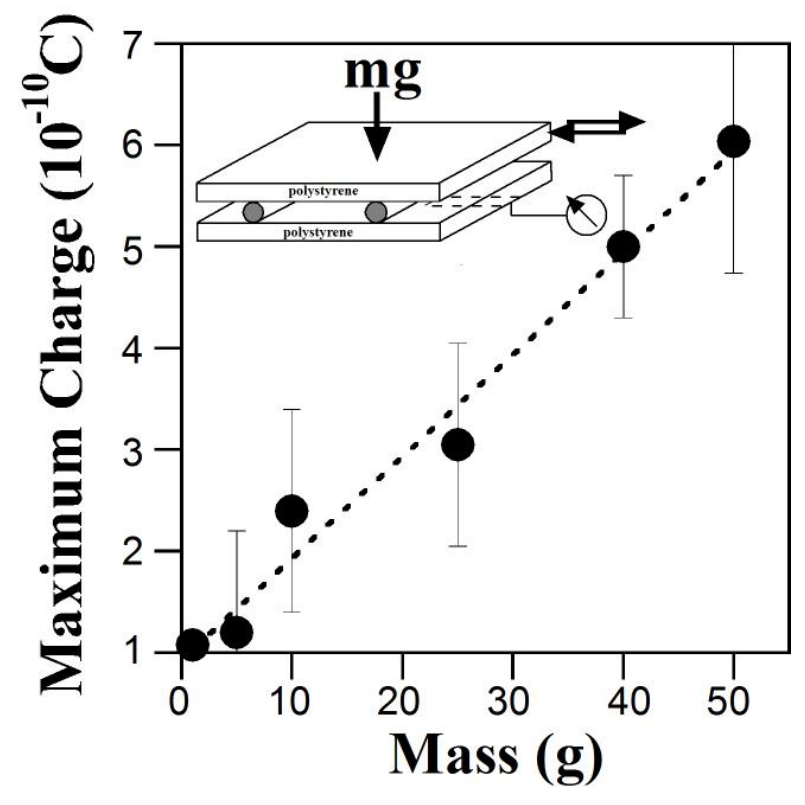

